\title{
Single Visit Replacement of Central Maxillary Using Fiber- Reinforced Composite Resin
}

\author{
Abu-Hussein Muhamad ${ }^{1}$, Abdulgani Azzaldeen ${ }^{2}$, Watted Nezar ${ }^{3}$
}

\begin{abstract}
Fiber reinforced composites are high strength filling materials composed of conventional composites and glass fibres. They exhibit extensive applications in different fields of dentistry. This clinical report present a case where FRC technology was successfully used to restore central maxillary incisor edentulous area in terms of esthetic-cosmetic values and functionality.
\end{abstract}

Keywords: Case report, Composite resin, Fiber-reinforced composite, Resin-bonded fixed partial dentures

\section{Introduction}

Loss of anterior teeth is a common form of injury, particularly in children and adolescents. On the other hand side, elderly people who are retaining their teeth for longer period of time, have often advanced caries or periodontal diseases which may lead to extraction of teeth. Patients with lost anterior teeth require immediate attention for restoration of esthetic-cosmetic and functional reasons.[1] With increased patient demand for tissue maintenance and esthetic, as well as to reduce treatment costs, causes clinician to seek materials and techniques that enable minimally invasive and chairside (direct) fabrication on teeth replacement with fixed partial dentures (FPD).[1,2]

Over the last few years, the development of fiberreinforced composite (FRC) has offered the dental profession the possibility of fabricating resin-bonded, esthetically good and metal-free tooth restorations for single and multiple teeth replacement. FRC-fixed partial denture (FPD) could be an alternative to metal frame resin-bonded-FPD, and also to full-coverage-crown-retained FPD and implant supported crowns [3, 4]. FRC, made of glass fibers, is the only existing esthetically acceptable material, which can be processed in mouth to the shape of a framework of a bridge, simultaneously adhere to the remaining tooth substance, and reach the adequate strength in terms of biting function of human.[3,4,5]

Many studies have focused on improvement of FRC FPD's strength [5, 6]. The most accepted concept to fabricate FRC FPDs is based on the use of continuous unidirectional glass (bundle) fibers in dimethacrylatepolymethylmethacrylate resin matrix as a substructure for the FPD [7].With the FRC FPDs, there are two approaches on the use of the fibers: one is based on conventional tooth preparation and laboratorymade restorations, while the other is based on using the fibers in minimally invasive restoration (conservative) by direct or indirect fabrication. FRC systems enable the use of different retainer elements even in the same FPD (hybrid-type) [4].

There are many types of fibers available for reinforcement and each type has its own unique characteristics. For example glass fibers seem to be the fibers of choice in dental applications because of the good adhesion of silanated glass fibers to mono- and dimethacrylates [5,6,7] and because of good esthetic properties. In addition the light-polymerized FRC substructure retains a sticky oxygen-inhibited layer on its external surface that allows direct chemical bonding with the covering composite, and thereby eliminates the need for mechanical retention as would be needed with a metal substructure.a Therefore awareness of the advantages and limitations of each type of fiber will enable the clinician to select the best fiber for a particular clinical situation. Hence the clinician must understand the basic structure of these materials and the different types that are available. This article presents an overview of the basic principles and techniques for the use of commercially available fiber reinforced composites in dentistry to support their clinical use as an alternative to conventional material.[5,6,7]

This paper describes clinical case of chairside-(directly) made FRC FPDs, which was used according to the principles of minimal invasiveness.

\section{Case Report}

A 47-year-old male patient had a chief complaint of esthetics and cosmetics because of a gap of missing right central maxillary incisor, After discussion with the patient, it became clear that the placement of an implant for the replacement of missing right maxillary cental incisor was not possible due to high costs of the treatment. (Fig. 1,2). 


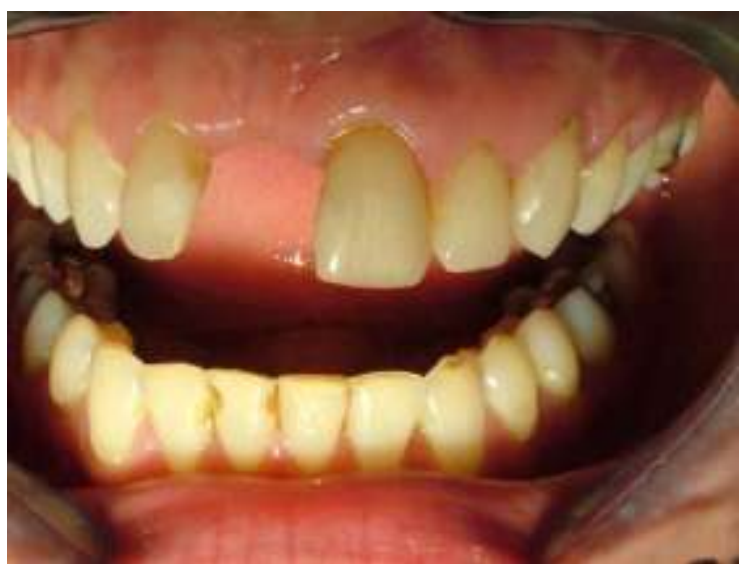

Fig1. Pre-operative intraoral With missing right central and limited space

The fabrication of a conventional fixed partial denture was avoided and refused from patient in order to conserve the remaining tooth substance. Option for the conventional treatment with implants or crown retained FPDs were remained open for the future. Directly made FRC FPDs was chosen in order to provide good esthetics and cosmetics, preserve tooth substance and postpone more invasive treatments. The treatment was completed during one appointment.

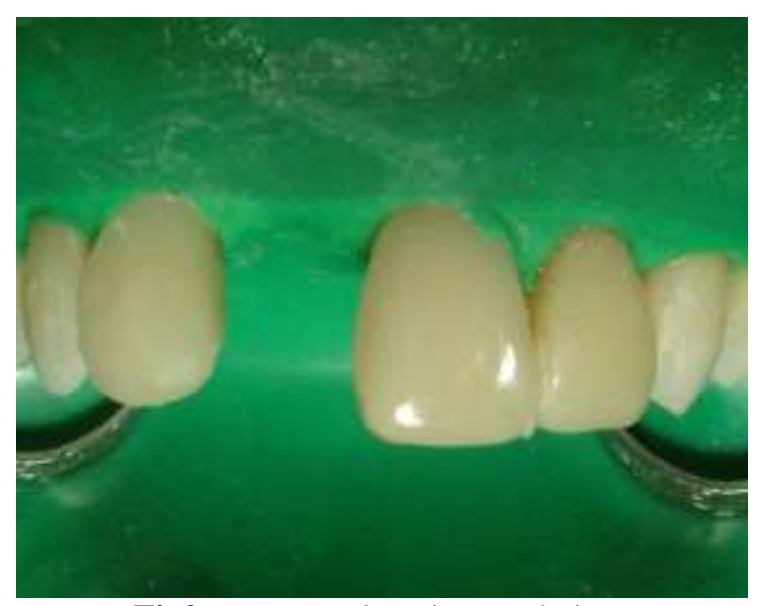

Fig2. Pre-operative intraoral view

There was free occlusal space on the palatal surface of central incisor for FRC framework to be placed. Consequently, no cavity preparation for receiving vertical support for the bridge was needed. Cotton roll for isolation was used although, the rubber dam is highly recommended.

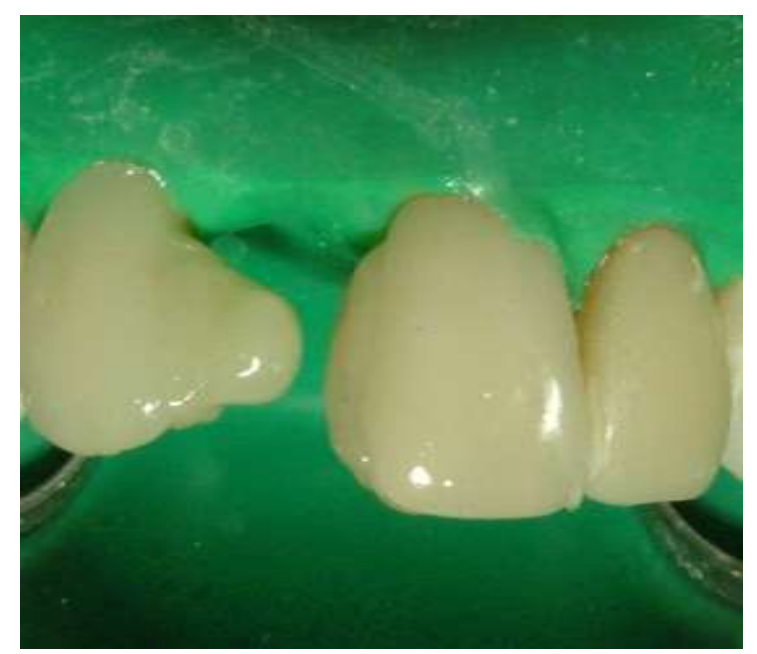

Fig3. Natural teeth bonded with 3M's Filtek Z250 A2 
A bundle of resin impregnated glass fibers (everStick Post, Stick Tech Ltd, Turku, Finland) was cut and spread from the ends for increasing the bonding surface area .

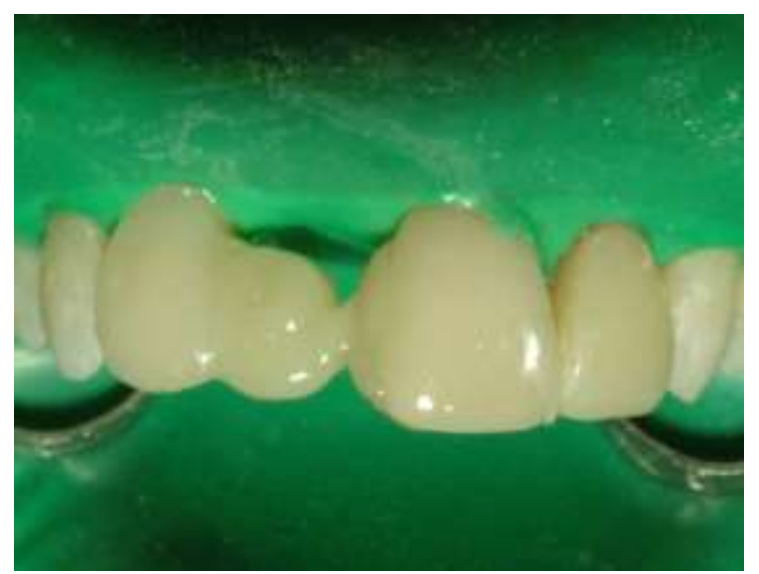

Fig4. Appearance of the FRC framework with a layer of flow

\section{composite between FRC and tooth}

The FRC framework was extended from the both buccal and palatal surfaces of right lateral incisor to palatal surface of central left incisor

After application of acid etching (37\% phosphoric acid gel), the gel was rinsed thoroughly and gently air dried. (Fig.3,4)

Adhesive resins were applied according to the manufacturer's instructions to tooth surface. Flowable composite resin (Stick Flow, Stick Teck Ltd, Turku, Finland)was applied on the bonding surfaces prior placing the resin Impregnated fibers (everStick). The flow composite was not light cured before fibers were pressed tightly against the tooth surface using transparent a silicone package (mold) of the fibers. The resin impregnated fibers were light cured initially through the silicone mold. (Fig. 5 )
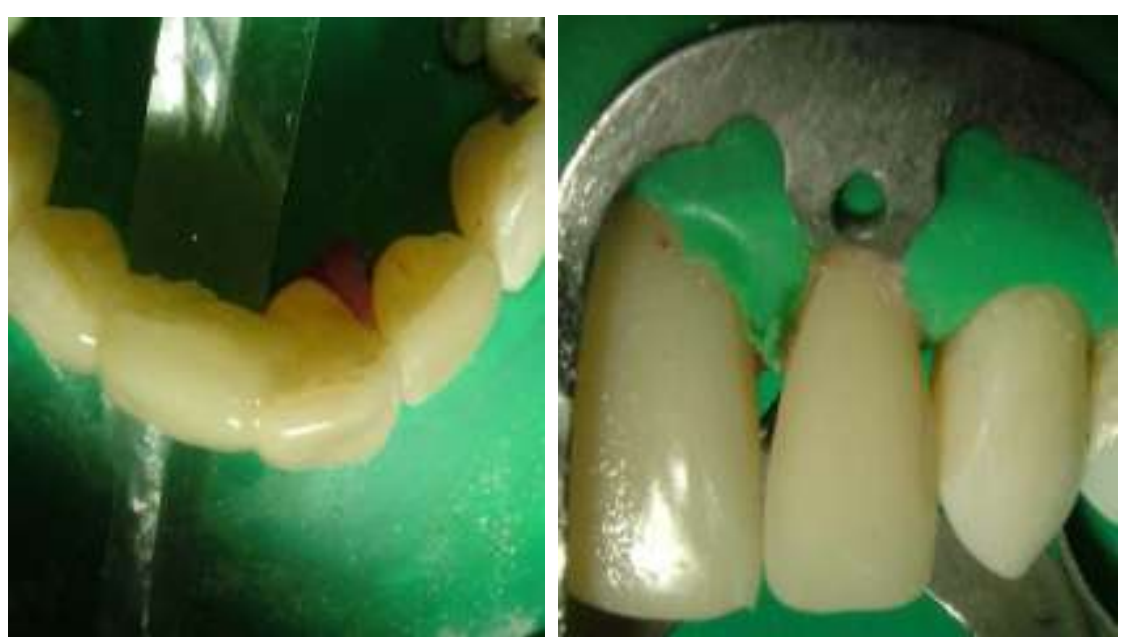

Fig5. Contoured strip on the gingival surface, Bonding lateral with Ivory $90 \mathrm{~N}$ clamp. Stiff clamp allows you to place margin under retracted gingiva.

The purpose of the flow composite was to seal the space between the fibers and the enamel surface. The fiber framework was polymerized two times for 40 seconds. Fiber framework was fully covered with a thin layer of flow composite resin and pontic wasbuild up by using hybrid type particulate filler composite resin. (Fig.6)Successful chemical bond between fiber framework and veneered composite was achieved by curing. The shade of final veneered composite resin was selected using composite shade guide and occlusion was carefully adjusted (Fig. 4). In this case canine guidance occlusion was avoided. The treatment outcome has been followed over 2 years without existence of any kind of serious problem. (Fig.7) 


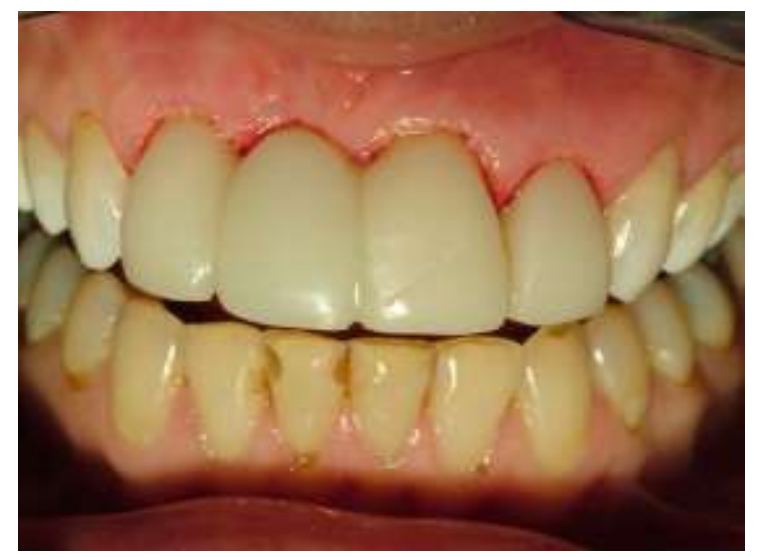

Fig6. Finished FPD

\section{Discussion}

Broken maxillary anterior teeth and replacing the missing or extracted maxillary anterior teeth in just one session is one of the most important esthetic problems faced by clinician as lead to improve the smile of their patients. $[8,9,10]$

Composite laminate, metal or all ceramic bridge work, dental implant and fiber reinforced composite bridges are all options in this case. The patient refused the dental implant because of high cost and long duration of treatment.[11,12,13] Fiber-reinforced composite bridges and all ceramic crowns might have been an alternative treatment but the possible need to prepare the teeth for fiber restoration and of course for the bridgework discouraged the patient and led me to construct the composite laminate restoration, this composite laminate restoration without preparation allowed the patient to see the appearance of her anterior teeth after treatment in just one sitting without any preparation. $[14,15,16,17]$
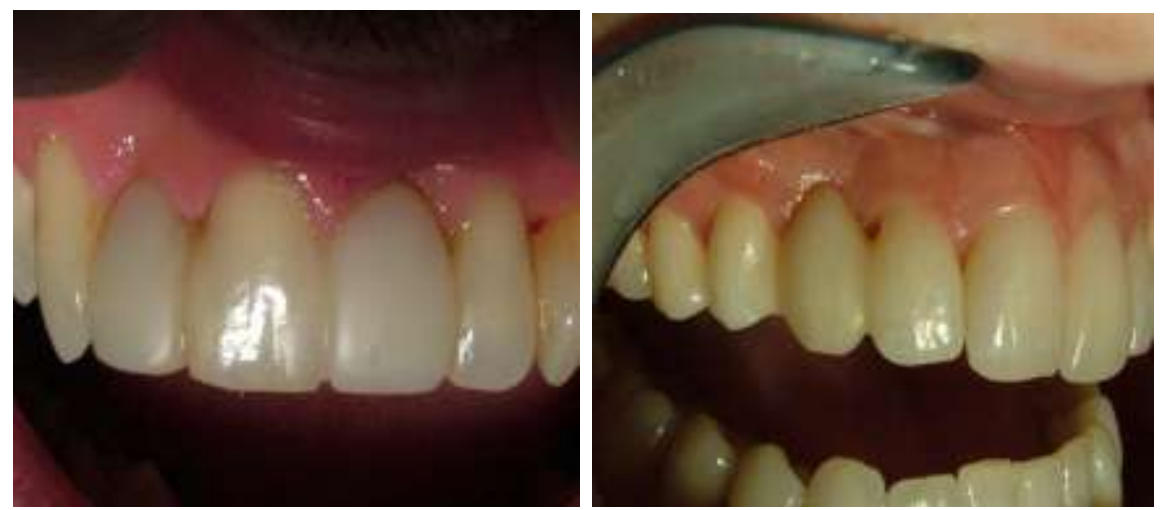

Fig7. Final view of the restoration having FRC framework veneered with filling composite resin, Lateral view of the final restoration

The advantage of direct laminate technique are that the restoration may be evaluated as reversible treatment procedure, can be repaired intro orally, can be done in just one session with amazing make over, and to my experience if high quality composite resin is used with right technique with high quality of polishing kit there will be no notable discoloration will be observed in years. [5,8,17]

The direct composite laminate technique has become more affective because of improvement in adhesive chemistry. Porcelain laminate veneer, metal ceramic crowns, and all ceramic crowns are expensive and need tooth preparation. $[5,17]$ These kinds of restorations also take a long time, they are irreversible, based on this knowledge, a direct composite laminate technique may be an important choice for treatment compared with other fixed dental prosthesis. Fiber-reinforced composite bridge can be a good alternative treatment. Resinbonded FRC FPDs are most commonly used in the anterior and premolar regions, rather than molar region, recent laboratory investigations have suggested that optimally designed FRC FPD made on nonprepared abutments can provide even higher load-bearing capacity for the FPD than conventional porcelain-fused tometal FPD can provide $[18,19]$. Thus, the development of the FRC materials and technologies may allow alternatives also for directly made molar replacements. $[15,19,20]$

The FRC framework is intended to be fully covered by veneering composite in order to obtain a polishable and tooth-coloured surface. Special attention needs to be paid to the interproximal regions. If the 
FRC framework is not properly covered by veneering composite, the darkness of the oral cavity can be transmitted through the connectors and can cause esthetic problems $[1,2,5]$.

The composition of the polymer matrix and fiber orientation has the major role in bonding ability and durability of veneered composite to the FRC framework or resin luting cement. It has been concluded that preimpregnation of the fibers with the light-polymerizable dimethacrylate resin system containing linear polymer phases is of importance to optimize the interfacial adhesion of FRC framework to composite veneer. Using a combination of dimethacrylate monomer resin and linear polymer, which forms semiinterpenetrating polymer network (semi-IPN) after being polymerized, offers better bonding site for veneered composite by means of interdiffusion bonding [ 18-20]. Recent laboratory studies showed that bond strength of directly fabricated FRC FPD to the tooth surface is as good as particulate filler composite [15].

The development of dentin adhesive systems has also led to similar and minimally invasive preparations. But clinical longevity of these prosthesis was found to be poor due to lack of interaction between metals and composite resins, leading to detachment under occlusal forces [5,6]. In vitro studies have shown that FCR materials exhibit increased strength when compared to particulate resin alone and can withstand occlusal forces in load bearing situations. Vallittu and Sevelius studied clinical success of FRCPs and found 93\% survival rate after 24 months follow up. In another study Vallittu et al, showed success rate was to increase from $75 \%$ to $95 \%$ at 42 months [1 ].

Metal framework adhesive fixed prostheses in comparison were found to have $61 \%$ survival rate in long term follow up to 11 years. Corrente \& Hoppner et al et al studied resin-bonded fixed partial dentures and splints in periodontally compromised patients and the 20 year cumulative survival rate from life table analysis was $76.2 \%$ ( $70.6 \%$ for fixed partial denture and $80.7 \%$ for splints).[21]

\section{Factors influencing success rate:}

a) The operator experience has been considered a significant factor to influence the success rate [22]

b) A well-designed preparation is another significant factor on resin-bonded FPD retention.[22]

c) Another aspect is the potential reinforcement provided for polyethylene fibers.[1] The adhesion between the fiber and the composite could increase the resistance and the hardness of the material allowing deflection without fracture.[23]

d) The prosthetic space in resin-bonded FPDs is a significant factor to determine the treatment success. The distance should not be larger than $15 \mathrm{~mm}$, because the FPD would suffer a higher deflection and could fail. A large prosthetic space in the mandible might increase the failure rate in 3 times .[24]

e) The use of additional polyethylene strips in the fabrication of the FPD and micro etching of the internal interface with aluminum oxide can also increase the resistance and mechanical adhesion with the composite resin, respectively.[21]

This clinical report describes the aesthetic replacement of a missing central maxillary left incisor and splinting of periodontally compromised teeth adjacent to the prosthetic space with a conservative FRC-FPD resulting in success over a short-term follow up. This treatment option can be categorized as a periodontal prosthesis [5]. Direct technique is conservative, cost effective, eliminates laboratory procedure. The prosthesis can be placed in a single visit using natural teeth, acrylic tooth or composite resin teeth as a pontic. The aesthetics of the FRC-FPD was shown to be considerably better than the aesthetics of FPDs with metal frameworks, as subjectively determined by many observers $[14,15]$.

The use of composites to build primary teeth provides a vital final aspect, withnatural opalescence, translucency and opacity. [5] This ensured proper esthetics and predictable bondability with adjacent teeth. Insta Fibre Splint was selected in the present case, as it is an ultrahigh molecular weight polyethylene fiber having virtually no memory; hence, it adapts to the contours of the teeth and dental arch. [25]It is practically colorless and disappears within the composite or acrylic without showthrough offering excellent esthetics.[26] Key factors that influence the physical properties of fiber reinforced structures are fiber loading within the restoration and efficacy of the bond at the fiber resin interface, fiber orientation and fiber position in the restoration.[25,26]

Earlier evidence suggests the use of FRC as a successful alternative to conventional space maintainers over a short follow-up period. Long-term studies are required to evaluate their prolonged use.[5]

\section{Conclusion}

Fiber-reinforced composite FPDs can satisfy the expectations of patients who seek safe, biocompatible, affordable, and esthetic restorations. However, clinicians are restricted by factors such as type of preparation, fiber frame design, span length, and the resin composite or luting agent. Most of available literature includes short-term follow-up studies. The long-term behavior of FRC bridges needs to be assessed for better understanding of their performance. 


\section{References}

[1]. Vallittu PK, Sevelius C. Resin-bonded, glass fiber-reinforced composite fixed partial dentures: A clinical study. J Prosthet Dent 2000;84:413-18.

[2]. Butterworth C, Ellakwa AE, Shortall A. Fiber-reinforced composites in restorative dentistry. Dent Update 2003;30: 300-06.

[3]. P. K. Vallittu, "Flexural properties of acrylic resin polymers reinforced with unidirectional and woven glass fibers," The Journal of Prosthetic Dentistry, vol. 81, no. 3, pp. 318-326, 1999.

[4]. S. H. Kimand D. C. Watts, "Effect of glass-fiber reinforcement and water storage on fracture toughness (KIC) of polymerbased provisional crown and FPD materials," International Journal of Prosthodontics, vol. 17, no. 3, pp. 318-322, 2004

[5]. Nezar Watted,Abdulgani Azzaldeen ,Muhamad Abu-Hussein; AESTHETIC REPLACEMENT OF CONGENITALLY MISSING TOOTH USING FIBER-REINFORCED COMPOSITE (FRC) Int J Dent Health Sci 2014; 1(4): 644-653

[6]. R. V. Curtis and T. F. Watson, Dental Biomaterials: Imaging, Testing and Modelling, chapter 9, Woodhead Publishing Company, 2008, Valittu PK, Fiber-reinforced composites for dental applications.

[7]. S. R. Dyer, L. V. J. Lassila, P. Alander, and P. K. Vallittu, "Static strength of molar region direct technique glass fibrereinforced composite fixed partial dentures," Journal of Oral Rehabilitation2005, vol. 32, no. 5, pp. 351-357

[8]. Abu-Hussein M ., Watted N ., Abdulgani Azz ., Abu-Shilabayeh H. ; Anterior dental esthetics in primary teeth; International Journal of Public Health Research 2015;4(1),25-36

[9]. Abu-Hussein M, Abdulgani A, Watted N, Zahalka M ; Congenitally Missing Lateral Incisor with Orthodontics, Bone Grafting and Single-Tooth Implant: A Case Report. J Dent Med Sci 2015,14: 124-130.

[10]. Kulkarni G, Lau D, Hafezi S.Development and testing of fiber-reinforced composite space maintainers. J Dent Child. 2009; 76(3): 204-8.

[11]. Abu-Hussein M, Chlorokostas G, Watted N, Abdulgani A, Jabareen A ; Pre- Prosthetic Orthodontic Implant for Management of Congenitally Unerupted Lateral Incisors - A Case Report. J Dent Med Sci 2016,15: 99-104.

[12]. Abu-Hussein M, Watted N, Abdulgani A, Borbély ; Modern Treatment for Congenitally Missing Teeth: A Multidisciplinary Approach. Int J maxillofacial res2015, 1: 179-190.

[13]. Mai A, Azzaldeen A, Nezar W, Chlorokostas G, Muhamad AH ; Extraction and Immediate Implant Placement with SingleStageSurgical Procedure: Technical Notes and a Case Report. J Dent Med Sci 2016,15: 95-101.

[14]. Muhamad AH, Azzaldeen A, Nezar W, Mohammed Z ; Esthetic Evaluation of Implants Placed after Orthodontic Treatment in Patients with Congenitally Missing Lateral Incisors. J Adv Med Dent Scie Res2015, 3: 110-118.

[15]. Abdulgani M, Abdulgani Az, Abu-Hussein M ; Two Treatment Approaches for Missing Maxillary Lateral Incisors: A Case. J Dent Med Sci 2016,15: 78-85.

[16]. Abu-Hussein M, Watted N, Abdulgani A ; Managing congenitally missing lateral incisors with single tooth implants. Dent Oral Craniofac Res 2016,2: 318-324.

[17]. Abu-Hussein M, Watted N ;Maxillary Midline Diastema - Aetiology And Orthodontic Treatment- Clinical Review. J Dent Med Sci2016, 15: 116-130

[18]. Sebnem Begum Turker, Isil Damla Sener. Replacement of a maxillary central incisor using a polyethylene fiberreinforcedcomposite resin fixed

[19]. partial denture: A clinical report. Turkey J Prosthet Dent 2008;100:254-258.

[20]. Paul van Wijlen.A Modified Technique for Direct, Fibre-Reinforced, Resin-Bonded Bridges: Clinical Case Reports. J Can Dent Assoc 2000; 66:367-71.

[21]. Neslihan A, Ayca A. Fiber-reinforced technology in multidisciplinary chairside approaches. Idjr $2008 ; 19$ [3]: 272 -277.

[22]. Corrente G, Vergnano L, Re S, et al. Resin-bonded fixed partial dentures and splints in periodontally compromised patients: a 10year follow-up. Int J Periodontics Restorative Dent 2000 Dec; 20(6) :628-36.

[23]. Miller TE, Hakimzadeh F, Rudo DN. Immediate and indirect woven polyethylene ribbon reinforced periodontal -prosthetic splint: A case report. Quintessence Int 1995; 26:267-271.

[24]. Rudo DN, Karbhari VM. Physical behaviors of fiber reinforcement as applied to tooth stabilization. Dent Clin North Am 1999; 43:7-35.

[25]. Hoppner et al, Rehabilitation of periodontal compromised teeth with fiber reinforced composite resin- a case report. Quintessence Int 2011; 42: 113-120.

[26]. Ganesh M, Tandon S. Versatility of Ribbond in contemporary dental practice trends biomater. Artif Organs 2006;20:53-8.

[27]. Kirzıoglu Z, Erturk MS. Success of reinforced fiber material space maintainers. J Dent Child 2004;71:158-62. 